\title{
Retinal vessel diameters and reactivity in diabetes mellitus and/or cardiovascular disease
}

\author{
R. Heitmar1* (D, G. Y. H. Lip ${ }^{1,2}$, R. E. Ryder ${ }^{3}$ and A. D. Blann²
}

\begin{abstract}
Background: Retinal vessel calibre and vascular dilation/constriction in response to flicker light provocation may provide a measure distinguishing patients suffering from diabetes mellitus and/or cardiovascular disease.

Methods: One hundred and sixteen age and sex matched patients with diabetes mellitus (DM), cardiovascular disease (CVD) and both DM and CVD (DM + CVD) underwent systemic and intraocular pressure measurements. Retinal vessel calibres were assessed using a validated computer-based program to compute central retinal artery and vein equivalents (CRVE) from monochromatic retinal images. Vessel dilation and constriction responses to flicker light provocation were assessed by continuous retinal vessel diameter recordings. Plasma endothelial markers von Willebrand factor (vWf) and soluble E selectin (sEsel) were measured by ELISA.

Results: Retinal vessel calibres were comparable across groups but CRVE correlated significantly with disease duration in DM patients $(r=0.57, p<0.001)$. Patients suffering DM only exhibited reduced arterial vasomotion at rest and reduced arterial constriction following flicker light induced vessel dilation compared to patients with CVD and those suffering both CVD + DM ( $p=0.030)$. Patients suffering from CVD + DM exhibited significant differences between each flicker cycle in regards to arterial maximum constriction $(p=0.006)$ and time needed to reach arterial maximum dilation $(p=0.004)$, whereas the other two groups did not show such inconsistencies between individual flicker cycles. vWf was raised in CVD + DM compared to the other two groups ( $\leq \leq 0.02)$, whilst sEsel was raised in CVD + DM compared to DM alone $(p=0.044)$.
\end{abstract}

Conclusions: Dynamic retinal vascular calibres as obtained by continuous diameter measurements using flicker light provocation can reveal subtle differences between groups suffering from CVD with and without DM. This difference in reaction pattern and lack of arterial constriction in DM may provide a suitable marker to monitor progression.

Keywords: Diabetes mellitus, Cardiovascular disease, Retinal vessel diameter, Retinal vessel reactivity, Blood glucose

\section{Background}

Cardiovascular disease (CVD) has a complex pathogenesis and can manifest as coronary artery disease (such as previous myocardial infarction and coronary artery stenosis/occlusion) cerebrovascular disease (leading to stroke) and peripheral artery disease (often requiring amputation). Diabetes mellitus (DM) is a major risk factor for CVD, but many diabetics also have other risk

\footnotetext{
*Correspondence: r.heitmar1@aston.ac.uk

' School of Life and Health Sciences, Aston University, Aston Triangle, Birmingham B4 7ET, UK

Full list of author information is available at the end of the article
}

factors such as hypertension, and suffer from microvascular disease, such as of the retinal vessels, causing retinopathy $[1,2]$. Leading clinical aspects of retinal vascular disease are disease duration and the degree of hyperglycaemia (as assessed by glycated haemoglobin $\left[\mathrm{HbA}_{1 \mathrm{c}}\right]$ ), and accordingly the accurate assessment of retinal function is a highly-sought after clinical tool [3, 4].

The retinal vasculature is readily accessible and can be observed non-invasively by fundus photography, video recording and tomographic technology. It's potential as screening tool for cerebrovascular [5] and cardiovascular disease $[6,7]$ has been supported through large 
population studies [8-11]. These studies demonstrated a relationship between retinal vessel calibres and cardiovascular risk $[11,12]$. Individuals with good cardiovascular health are less likely to have signs of retinopathy such as dilated retinal venules and narrow retinal arterioles, both of which are associated with increased risk of stroke and coronary artery disease [13].

While retinal vessel calibres provide only static indices, dynamic measurements such as retinal vessel reactivity to flicker light provocation can provide further insight into the status of the retinal microcirculation. Several authors have demonstrated a link between measures of cardiovascular health and retinal vessel dynamics, such as a decrease in retinal vessel dilation in presence of decreased flow mediated dilation of the brachial artery [14], prolonged reaction times in retinal arterial responses to flicker light in patients suffering from coronary artery disease [15], and decreased vessel dilation to flicker light in patients with coronary artery disease, and which depends on the severity of the disease [16]. Previous research has shown reduced retinal vessel dilation to flicker light in DM patients compared to controls which further reduced with the severity of diabetic retinopathy [17-19]. This lack of reactive hyperaemia in DM patients has been attributed to abnormal glial regulation of the retinal vasculature [20] as well as endothelial cell dysfunction leading to reduced nitric oxide (NO) synthase and sensitivity $[17,21]$. While most studies conducted in DM patients examined both arteries and venous responses, they have not evaluated the full response to flicker light which includes the vasomotion at rest, vasoconstriction post flicker and reaction time.

Whilst retinal vessel dynamics have the potential as tool for risk stratification and screening as evidenced by the progressively decreasing vessel dilation with increasing severity of diabetic retinopathy, the relative influence of how different pathological combinations and risk factors impact on retinal vessel parameters is unclear. We hypothesised that retinal vessel responses to flicker light would be more adverse in those patients with both DM and CVD compared to patients with either DM or CVD alone.

\section{Methods}

The study was approved by the National Health Service Research Ethics Committee East Midlands-Leicester (Ref: 12/EM/0062) and the Aston University Ethics Committee and adhered to the Declaration of Helsinki. Patients were recruited from the cardiovascular rehabilitation unit, hypertensive and diabetic outpatient clinics at City Hospital (Birmingham, UK). All patients gave written informed consent. Three groups were defined prior to recruitment: patients suffering from diabetes mellitus (type 1 and type 2) but free from cardiovascular: patients suffering from cardiovascular disease but free from diabetes, and patients suffering from both diabetes and cardiovascular disease. Diabetes was defined by attendance at a diabetes clinic and $\mathrm{HbA}_{1 \mathrm{c}}>50 \mathrm{mmol} / \mathrm{mol}(6.7 \%)$. Cardiovascular disease was defined as history of myocardial infarction, coronary artery stenosis/occlusion, coronary artery bypass grafting, stroke, iliac/femoral artery stenosis or bypass or lower limb/foot/toe amputation. Exclusion criteria included recent ( $<3$ month) myocardial infarction, stroke, or surgery, cancer, autoimmune disease (such as rheumatoid arthritis) or ophthalmological disease such as age-related macular degeneration. Serum endothelial markers von Willebrand factor (vWf) and soluble E selectin (sEsel) were measured by commercial ELISA (Dako, Ely, UK and R \& D Systems, Abingdon, UK). Clinical, laboratory, medication and demographic data were collected and are shown in Table 1.

Patients were advised to abstain from caffeinated drinks for a minimum of $12 \mathrm{~h}$ prior to their ocular examination. All patients were seated in a temperature-controlled room $\left(21 \pm 2{ }^{\circ} \mathrm{C}\right)$ for a minimum of $15-20 \mathrm{~min}$ to achieve a stable blood pressure (systolic blood pressure [SBP] and diastolic blood pressure [DBP] were measured using a digital sphygmomanometer [UA767, PMS Instruments, UK]). Intraocular pressure (IOP) was measured using a rebound $t$ tonometer (I-Care, Mainline Instruments Ltd., UK) after which pupils were dilated with $1 \%$ tropicamide (Chauvin Pharmaceuticals Ltd., Kingston-Upon-Thames, UK).

\section{Dynamic and static retinal vessel assessment}

Once pupils were fully dilated, digital fundus images and reactivity parameters of retinal arteries and veins to flicker light provocation were obtained from one eye of each patient [58 right eyes and 58 left eyes] (Retinal vessel analyser [RVA], Imedos Systems [UG] haftungsbeschraenkt Jena, Germany) [22]. For static vessel analysis monochromatic (red-free) fundus images were obtained, at a $30^{\circ} \mathrm{C}$ camera angle with the optic nerve head $(\mathrm{ONH})$ centred, using the inbuilt Zeiss FF450 + fundus camera (Zeiss GmbH, Germany), see Fig. 1. Summarised retinal vessel calibres of retinal arteries (central retinal artery equivalent, CRAE), veins (central retinal vein equivalent, CRVE) and the retinal artery-to-vein ratio (AVR) were calculated according to a standard protocol [23].

Dynamic imaging was followed by static imaging. Retinal vessel diameters were measured continuously at a sampling rate of $25 \mathrm{~Hz}$. Stimulation of retinal blood vessels was done by optoelectronic interruption of the green fundus illumination used by the RVA resulting in a flicker light provocation with a $12.5 \mathrm{~Hz}$ frequency [24-26]. After BP stabilisation and image focussing one vessel segment 
Table 1 Clinical data, medication and demographics

\begin{tabular}{|c|c|c|c|}
\hline & $\mathrm{DM}(\mathrm{n}=36)$ & $\operatorname{CVD}(n=43)$ & $\begin{array}{l}\text { DM and CVD } \\
(\mathrm{n}=37)\end{array}$ \\
\hline DM type: 1/2 & $2 / 34$ & - & $1 / 36$ \\
\hline Ethnicity: CA/SA/B & $28 / 4 / 4$ & $37 / 4 / 2$ & $32 / 5 / 0$ \\
\hline Age (years) & $64(10)$ & $64(11)$ & $65(9)$ \\
\hline Gender (m/f) & $24 / 12$ & $30 / 13$ & $32 / 5$ \\
\hline SBP (mmHg) & $130(13)$ & $125(19)$ & $125(16)$ \\
\hline $\mathrm{DBP}(\mathrm{mmHg})$ & $75(9)$ & $75(13)$ & $67(10)^{*}$ \\
\hline $\begin{array}{l}\text { HR (beats per } \\
\text { minute) }\end{array}$ & $76(14)$ & $67(11)^{*}$ & $72(16)$ \\
\hline IOP $(\mathrm{mmHg})$ & $15(3)^{+}$ & $13(2)$ & $14(2)$ \\
\hline BMI $\left(\mathrm{kg} / \mathrm{m}^{2}\right)$ & $31(6)$ & $27(4)^{*}$ & $31(6)$ \\
\hline $\mathrm{HbA1c}(\mathrm{mmol} / \mathrm{mol})$ & $58(15)$ & $42(3)^{*}$ & $61(20)$ \\
\hline DM duration (years) & $11(4.5-16)$ & - & $10(4-17)$ \\
\hline \multicolumn{4}{|l|}{ Medications } \\
\hline $\begin{array}{l}\text { Calcium channel } \\
\text { blocker }\end{array}$ & $20,56 \%$ & $13,30 \%$ & $14,38 \%$ \\
\hline ACEI/ARB & $23,64 \%$ & $31,72 \%$ & $30,81 \%$ \\
\hline Metformin & $26,72 \%$ & - & $21,57 \%$ \\
\hline Sulphonylurea & $7,19 \%$ & - & $7,19 \%$ \\
\hline DPP-4 inhibitor & $8,22 \%$ & - & $6,16 \%$ \\
\hline Insulin & $15,42 \%$ & - & $14,38 \%$ \\
\hline GLP-1 agonist & $4,11 \%$ & - & $4,11 \%$ \\
\hline Piaglitazone & $4,11 \%$ & & $2,5 \%$ \\
\hline Lipid-lowering & $26,72 \%$ & $41,95 \%$ & $36,97 \%$ \\
\hline Aspirin & $11,31 \%$ & $35,81 \%$ & $30,81 \%$ \\
\hline Clopidogrel & $1,3 \%$ & $7,16 \%$ & $7,19 \%$ \\
\hline Nitrate & - & $11,26 \%$ & $11,30 \%$ \\
\hline Oral anticoagulant & $6,17 \%$ & $5,12 \%$ & $5,14 \%$ \\
\hline Beta-blocker & $10,28 \%$ & $19,44 \%$ & $22,59 \%$ \\
\hline Diuretic & $18,50 \%$ & $9,21 \%$ & $18,49 \%$ \\
\hline Thyroxine & $6,17 \%$ & $6,14 \%$ & $3,8 \%$ \\
\hline \multicolumn{4}{|c|}{ Cardiovascular disease } \\
\hline $\begin{array}{l}\text { Coronary artery } \\
\text { disease }\end{array}$ & - & $31,72 \%$ & $30,81 \%$ \\
\hline $\begin{array}{l}\text { Peripheral artery } \\
\text { disease }\end{array}$ & - & $5,12 \%$ & $4,11 \%$ \\
\hline $\begin{array}{l}\text { Cerebrovascular } \\
\text { disease }\end{array}$ & - & $8,19 \%$ & $3,8 \%$ \\
\hline
\end{tabular}

Data presented as mean with standard deviation, or as number of subjects and percentage

$C A$ caucasian, $S A$ South Asian, $B$ black, $M$ male, $f$ female, $S B P$ systolic blood pressure, $D B P$ diastolic blood pressure, $H R$ heart rate, $I O P$ intraocular pressure, $B M I$ body mass index, $H b A 1 C$ glycated haemoglobin, DM diabetes mellitus, $A C E l$ angiotensin-converting-enzyme inhibitor, $A R B$ angiotensin receptor blockers, DPP-4 dipeptidyl peptidase-4 ('gliptins'), GLP-1 glucagon-like peptide-1 (exenatide, liraglutide)

* Different compared to the two other groups $(p<0.01)$

+ Different to the CVD group $(p<0.01)$

of each, the superior temporal retinal artery and vein was chosen at a distance of 1.5-2 DD away from the margins of the $\mathrm{ONH}$. Baseline diameters of both the artery and vein were recorded according to the standard RVA protocol [26] for $50 \mathrm{~s}$ and then followed by three cycles of $20 \mathrm{~s}$ flicker provocation with each 80 s recovery time. Resulting in a $350 \mathrm{~s}$ measuring period during which the fellow eye was occluded to improve patient fixation. From these diameter recordings of arteries and veins the values for baseline diameter fluctuation (BDF) maximum dilation (MD), maximum constriction (MC) and dilation amplitude (DA), time to reach MD following flickering light initiation (reaction time, RT) and arterial constriction time $(\mathrm{CT}=$ time to reach $\mathrm{MC}$ ) were calculated for single flicker cycles and averaged responses [27].

Area under the curve parameters of retinal arteriolar responses to FL were calculated using the averaged time course of all three FL cycles. $\mathrm{AUC}_{\text {Baseline: }}$ area under the curve of the averaged $30 \mathrm{~s}$ prior to flicker start, $\mathrm{AUC}_{20 \mathrm{~s}}$ $\mathrm{FL}_{\mathrm{L}}$ : area under the curve of the averaged profile during $20 \mathrm{~s}$ flicker [28], $\mathrm{AUC}_{20}$ s Constriction: area under the curve of $20 \mathrm{~s}$ duration $10 \mathrm{~s}$ following flicker cessation and $\mathrm{AUC}_{\text {Last } 30 \mathrm{~s}}$ : area under the curve of the last $30 \mathrm{~s}$ of the averaged reaction profile [29] (see Fig. 2).

\section{Statistical analysis}

We tested the hypothesis that levels of a test statistic with a mean of 100 arbitrary units (standard deviation 14 units) would differ by $10 \%$ in patients with diabetes and cardiovascular disease compared with those patients with diabetes alone or cardiovascular disease alone. To achieve this at alpha $<0.01$ and 1-beta $=0.80$ calls for data from 36 subjects per group. We therefore recruited consecutive patients until this sample size was achieved in each group. Associations between continuously-variable factors were sought using Spearman's correlation. With this sample size per group, risks of types 1 and 2 statistical error are minimised when a correlation coefficient $(\mathrm{r})$ exceeds 0.5 .

Cross-sectional differences between the three groups were assessed using analysis of variance or the KruskalWallis test, depending on distribution. Categorical data was analysed by the Chi squared test. Between group differences were sought using Tukey's post hoc test. Prior to averaging retinal vessel responses of the three flicker cycles, within group analyses of the three flicker light cycles were sought using Friedman's repeated (two-way) measures ANOVA. Comparisons between software generated dilatory parameters (the software generates three arterial parameters from averaged flicker responses $\left[\mathrm{A}_{\max }, \mathrm{A}_{\min }\right.$ and $\left.\mathrm{A}_{\text {peak }}\right]$ and one venous response $\left[\mathrm{V}_{\max }\right]$ ) and sequential diameter response analyses (SDRA) [27] were sought by paired $t$ test whereas subgroup analyses of patients with diabetic retinopathy were compared using the Mann-Whitney-U test or t test (supplementary data). Statistical significance was set at $\mathrm{p}<0.05$, and 


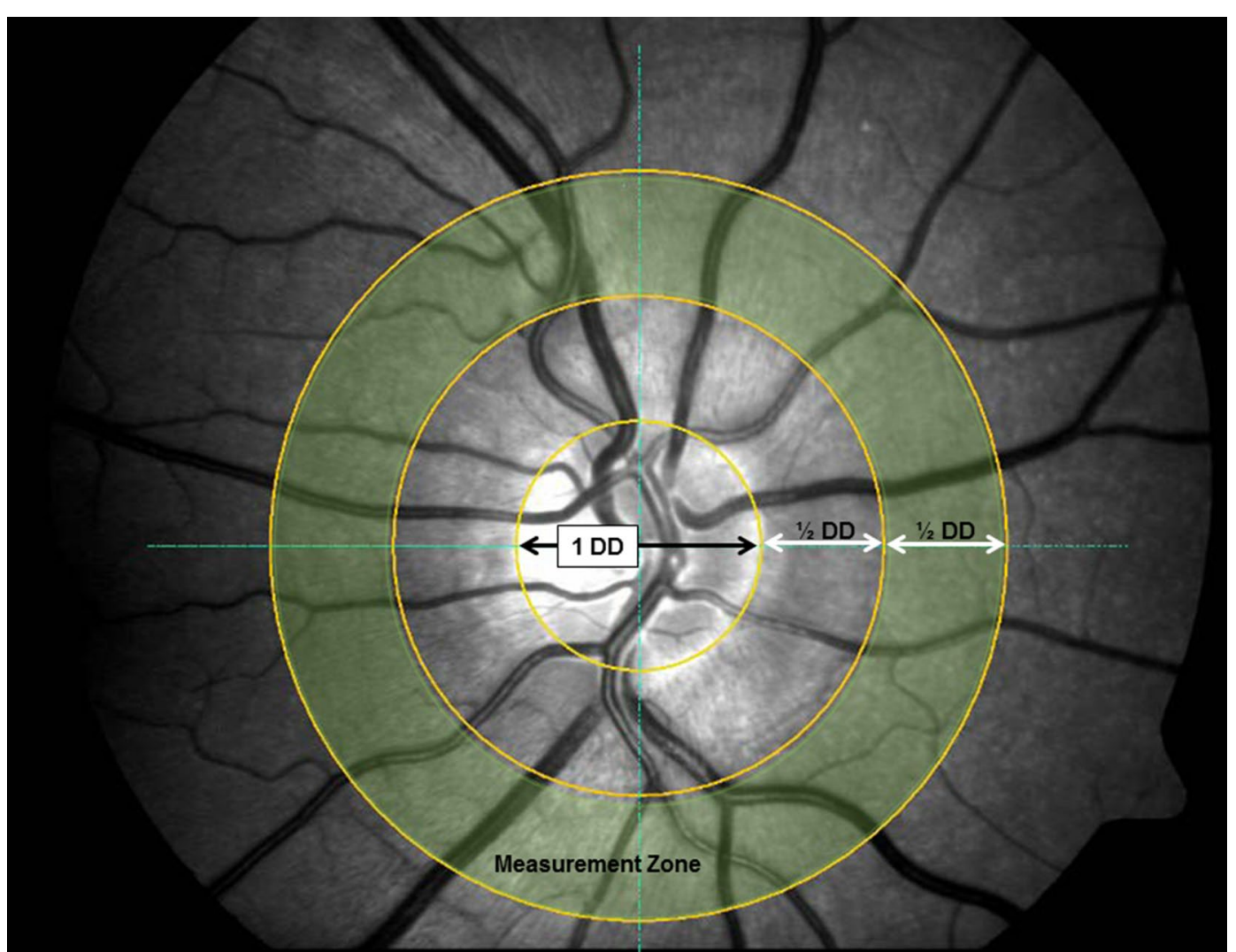

Fig. 1 Sample image showing measurement area for static retinal vessel evaluation. Central ring denotes the diameter of the disc ( 1 DD), the second and third circle are each $1 \frac{2}{2}$ DD separated to create a $1 / 2$ DD wide concentric ring segment around the optic nerve head. This ring segment is highlighted here in yellow to illustrate the area in which retinal arterial and venous calibres are measured to calculate central retinal artery and vein equivalents (CRAE and CRVE)

results are reported as either mean (standard deviation) or as median (upper quartile-lower quartile). All data were analysed using Statistica Version 12.4 (Dell Software, Dublin, Ireland).

\section{Results}

Table 1 shows clinical, demographic, medication and comorbidity details of the 116 patients (36 DM only [2 Type 1 and 34 Type 2], 43 CVD only and 37 CVD and DM [1 Type 1 and 36 Type 2]). The three groups were matched for age (ANOVA $\mathrm{p}=0.769)$ and $\operatorname{sex}\left(X^{2} \mathrm{p}=0.110\right)$. Patients with DM and CVD had lower DBP than the other two groups, whilst heart rate, HbA1c and body mass index were lower in those with CVD alone. There was no difference in the distribution of the type of CVD between the two groups with this disease $\left(X^{2} \mathrm{p}=0.405\right)$. Table 2 shows retinal indices (summarized retinal vessel calibres and software generated vessel dynamic retinal vessel parameters). There were no significant differences between the three groups.
Table 3 and Fig. 3 shows the averaged dynamic retinal vessel diameter and responses to flicker light stimulation of both arteries and veins. In cross-sectional analyses, the arterial BDF was lower in DM only group compared to the other two groups. The DM + CVD groups' arterial maximum constriction following flicker cessation was inconsistent throughout the three flicker provocations, with the second cycles' response being the largest compared to the first and third $(-1.79 ;-2.50$ and $-1.68 \% ; \mathrm{p}=0.006)$, this was not the case for the other two groups. The average arterial MC however was lower in those with DM alone compared to the two other groups. In single flicker analyses, the arterial RT was shortest in the second cycle than in the first and third cycles in the DM and CVD group only $(20 \mathrm{~s}, 16 \mathrm{~s}, 23 \mathrm{~s} ; \mathrm{p}=0.004)$. There were no such differences between individual flicker cycles in the other two groups.

There was no difference between the three groups in regards of the venous responses to flicker light provocation. Table 4 shows area under the curve data: this index confirmed the significantly lower arterial vessel 


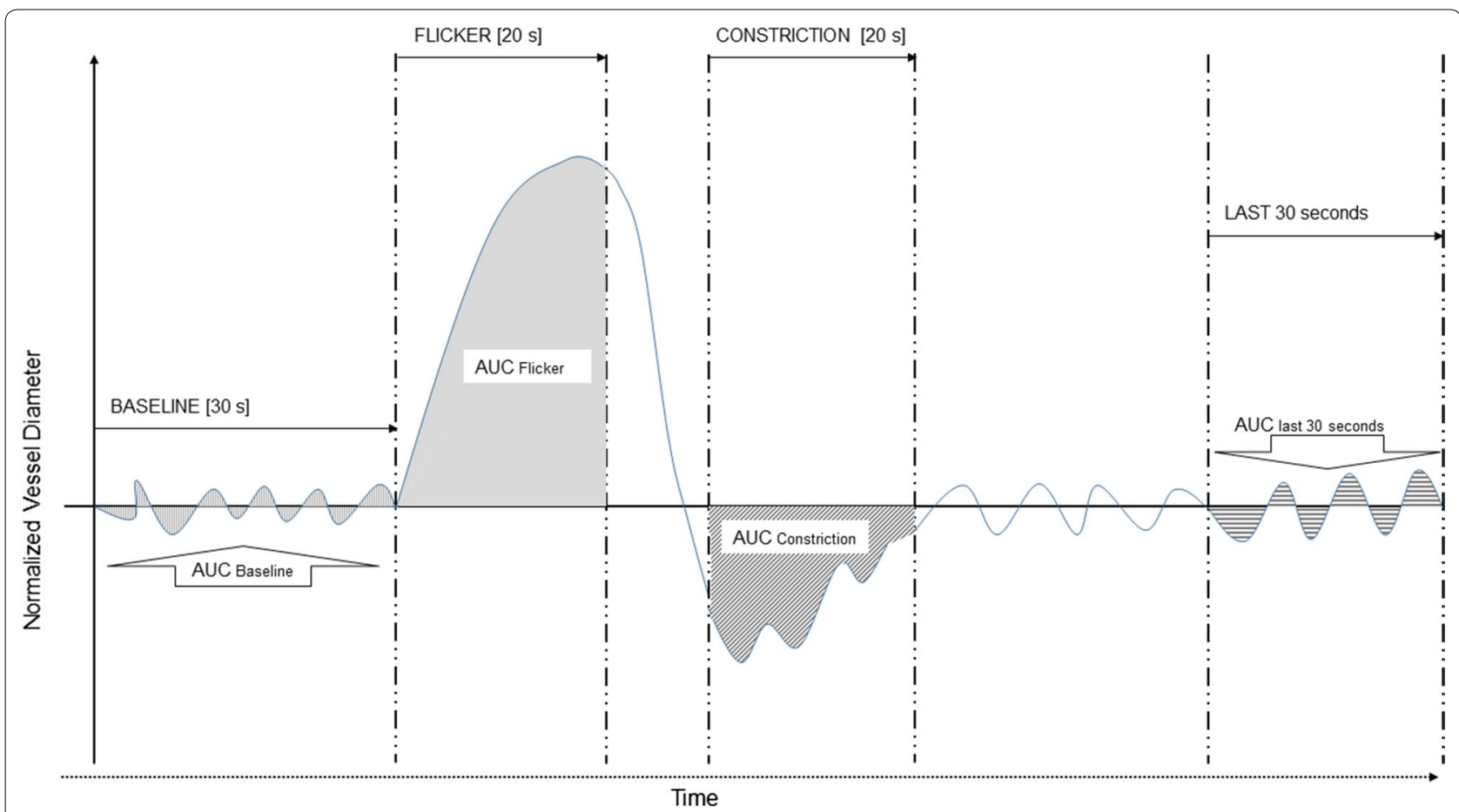

Fig. 2 Schematic to illustrate area under the curve calculations. The highlighted areas in grey denote the time zones from which "Area under the curve" (AUC) data was calculated for baseline, during flicker, constriction and of the final $30 \mathrm{~s}$

Table 2 Retinal parameters

\begin{tabular}{lcccc}
\hline & DM $(\mathbf{n}=\mathbf{3 6})$ & CVD $(\mathbf{n}=\mathbf{4 3})$ & DM and CVD (n= 37) & $\mathbf{p}$ value \\
\hline CRAE (au) & $178(21)$ & $175(18)$ & $175(11)$ & 0.660 \\
CRVE (au) & $213(19)$ & $208(19)$ & $0.83(0.01)$ & 0.212 \\
AVR & $0.84(0.02)$ & $0.85(0.02)$ & $0.90(0.10-1.80)$ & 0.389 \\
$A_{\text {max }}(\%)$ & $0.90(0.45-2.45)$ & $0.80(-0.1$ to 2.50$)$ & $-0.50(-1.10$ to 0.10$)$ & 0.558 \\
$A_{\text {min }}(\%)$ & $-0.40(-0.85$ to 0.05$)$ & $-0.50(-1.10$ to 0.20$)$ & $1.20(0.20-2.40)$ & 0.905 \\
$A_{\text {peak }}(\%)$ & $1.55(0.40-3.25)$ & $1.60(0.30-2.90)$ & $2.50(1.30-3.60)$ & 0.662 \\
$V_{\text {max }}(\%)$ & $3.25(2.00-3.90)$ & $3.10(1.80-4.10)$ & & 0.206 \\
\hline
\end{tabular}

Summarized retinal vessel calibres and software generated vessel reactivity parameters Data presented as mean (SD) or median (lower quartile-upper quartile)

Au arbitrary units, CRAE central retinal artery equivalent, CRVE central retinal vein equivalent, $A V R$ arterio-venous ratio, $A_{\max }$ IMEDOS software generated arterial maximum dilation to flicker, $A_{\min }$ IMEDOS software generated arterial maximum constriction following flicker, $A_{\text {peak }}$ IMEDOS software generated arterial dilation amplitude, $V_{\max }$ IMEDOS software generated venous maximum dilation to flicker

$p$ values by analysis of variance or the Kruskal-Wallis test as distribution demands

constriction in those with DM alone compared to the two other groups. The only significant correlation where the $r$ value exceeded 0.45 was between the disease duration in patients with diabetes alone and the CRVE $(\mathrm{r}=0.57$, $\mathrm{p}<0.001$ ). Of the 73 patients with diabetes, six had diabetic retinopathy (according to UK DR grading ${ }^{\mathrm{a}}$ ). With this small number, no analyses will be meaningful. Table 5 shows serum endothelial markers. vWf was higher in
$\mathrm{DM}+\mathrm{CVD}$ than in the other two groups, whilst sEsel was high in patients with DM + CVD versus patients with $\mathrm{DM}$ alone. In the DM alone group, vWf correlated with the venous reaction time in the first flicker cycle $(\mathrm{r}=0.5$, $\mathrm{p}=0.003)$. In the DM + CVD group, sEsel correlated with CRVE $(r=0.54, p=0.004)$ and with the AVR $(r=-0.62$, $\mathrm{p}=0.001)$. There were no other statistically significant correlations. 
Table 3 Dynamic arterial and venous vessel parameters

\begin{tabular}{|c|c|c|c|c|}
\hline & $\mathrm{DM}(\mathrm{n}=36)$ & CVD $(n=43)$ & $\mathrm{DM}$ and CVD $(\mathrm{n}=37)$ & p value \\
\hline \multicolumn{5}{|c|}{ Arterial parameters } \\
\hline$A_{S I Z E}(\mu m)$ & $110(16)$ & $113(20)$ & $116(18)$ & 0.294 \\
\hline BDF (\%) & $1.66(0.81)^{*}$ & $2.59(1.95)$ & $2.38(1.38)$ & 0.019 \\
\hline MD (\%) & $2.99(2.23)$ & $2.88(2.29)$ & $2.60(1.68)$ & 0.705 \\
\hline MC (\%) & $-1.45(0.87)^{* *}$ & $-2.06(1.50)$ & $-2.17(1.52)$ & 0.030 \\
\hline $\mathrm{RT}(\mathrm{s})$ & $18(11)$ & $20(12)$ & $20(12)$ & 0.679 \\
\hline$C T(s)$ & $40(10)$ & $38(8)$ & $40(7)$ & 0.531 \\
\hline \multicolumn{5}{|c|}{ Venous parameters } \\
\hline$V_{\text {size }}(\mu m)$ & $141(20)$ & $141(19)$ & $142(18)$ & 0.927 \\
\hline BDF (\%) & $1.91(0.88)$ & $2.53(2.09)$ & $2.45(1.52)$ & 0.192 \\
\hline MD (\%) & $4.34(1.85)$ & $4.28(1.96)$ & $4.16(2.24)$ & 0.928 \\
\hline MC (\%) & $-0.80(1.20)$ & $-0.79(1.53)$ & $-0.72(1.15)$ & 0.963 \\
\hline DA (\%) & $5.14(2.25)$ & $5.07(2.22)$ & $4.88(2.64)$ & 0.892 \\
\hline $\mathrm{RT}(\mathrm{s})$ & $21(9)$ & $20(4)$ & $21(7)$ & 0.972 \\
\hline
\end{tabular}

Retinal vessel reactivity parameters as calculated using sequential diameter response analysis (SDRA)

Data presented as mean (standard deviation) or median (inter quartile range)

All values given in percentage change to baseline vessel diameters or times in seconds

$B D F$ baseline diameter fluctuation, MD maximum dilation, $M C$ maximum constriction, $D A$ dilation amplitude, $R T$ reaction time, $C T$ constriction time, $s$ seconds

$p$ values by analysis of variance (ANOVA)

* Different compared to CVD group $(p<0.05)$

** Different compared to CVD and DM group $(p<0.05)$

\section{Discussion}

We report several differences in retinal vessel physiology in those with DM alone, those with CVD alone, and those with both DM and CVD which confirm and extend those of others [16-18]. However, in our study, we found no differences in CRAE, CRVE, the AVR or IMEDOS indices in those with or without diabetes and/or cardiovascular disease. The reason for this negative finding could be that early changes due to inflammation as described by Virchow's triad are present in all three groups. These alterations of the vascular system affecting blood flow, contributing to hypercoagulability and inducing vessel wall alterations through endothelial injury all contribute to an altered vessel structure over time but may present first by a change in vessel physiology prior to signs of structural damage.

Nevertheless, we found multiple differences to the responses of arteries to flicker stimulation, the most marked being those of the arterial MC and RT. These shared the property that responses with a difference in the second cycle compared to the first and third cycles in those with both DM and CVD, providing evidence of differences in the time course of arterial vessel dynamics in these pathologies. Averaged vessel diameter responses (as calculated by the software of the RVA and referred to as IMEDOS values) can mask differences not only in regards to reaction patterns but also underestimate the overall dilatory capacity [27]. While all three groups showed comparable results for summarised retinal vessel diameters and dynamic responses to flicker light patients suffering from DM alone exhibited increased vessel diameters (CRVE) with increased disease duration, confirming results of previous studies [30]. Furthermore, venous response to flicker light provocation was decreased with increasing disease duration. This decrease in venous dilation is in agreement with data described elsewhere $[17,18,21,30]$ and can be partly explained by the possibility that a pre-dilated vessel (as reflected by the increase in CRVE with increasing disease duration) has reduced residual dilatory capacity.

Area under the curve (AUC) analyses provides data that takes into account the time course as well as the extent of the vessel dilation recorded. The results of which confirm a lack of constriction in patients suffering from DM only compared to both other groups. Our finding is supported by a recently published study from Lott and colleagues, who have shown an impaired coronary and retinal arterial (and venous) vasoconstrictor response in patients suffering from DM compared to controls [31]. While this was a much smaller sample and applied a different methodology using a hyperoxic vs our flicker light stimulus, it confirms our results of an impaired ability of DM only patients to constrict to a physiologic stimulus. Our results of the SDRA and AUC 


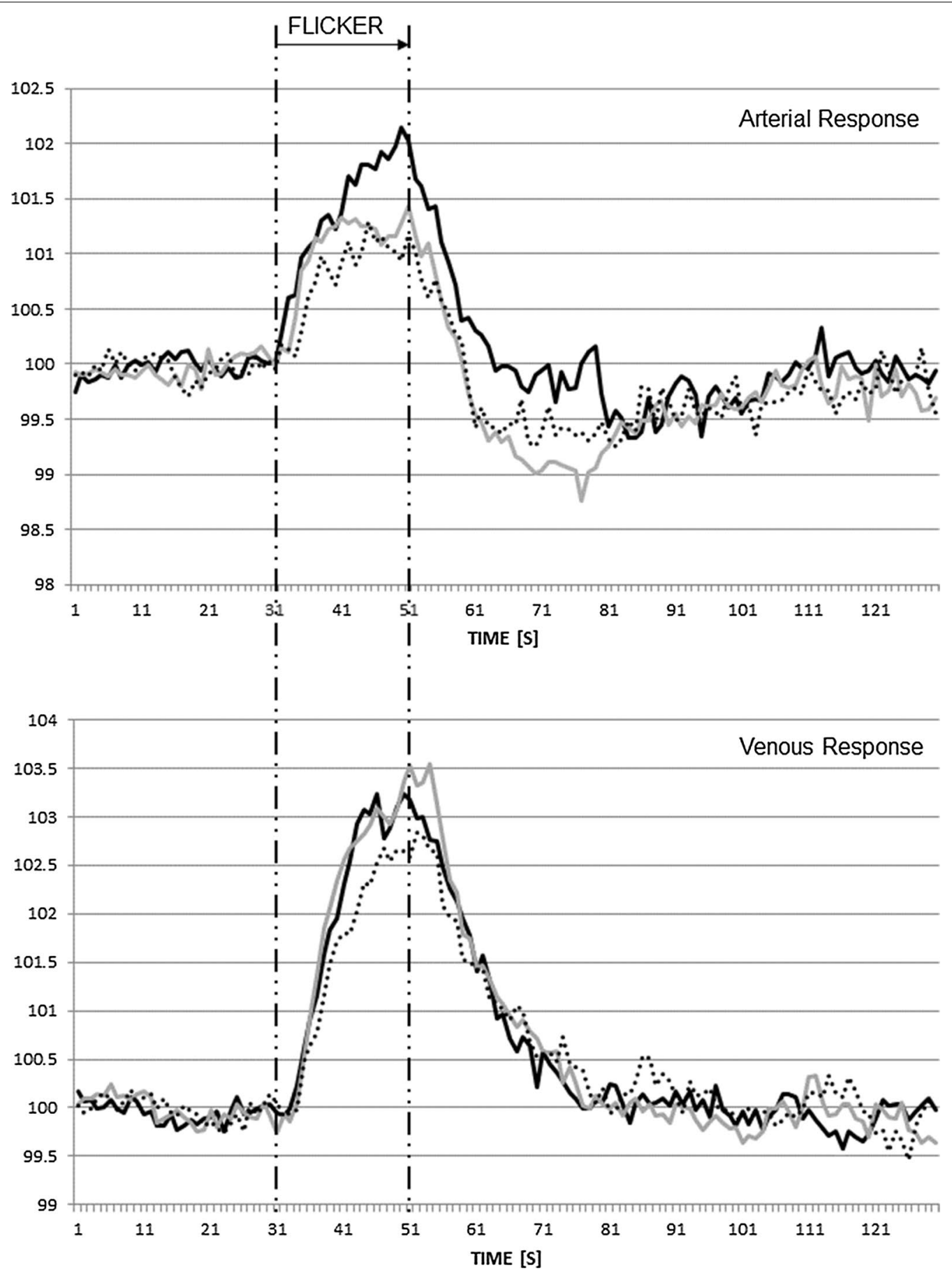

Fig. 3 Averaged response to flicker light provocation of retinal vessels. Mean arterial (top graph) and retinal vein (bottom graph) responses for all three patient groups (black solid line DM only; grey solid line CVD; black dotted line DM and CVD)

analysis confirm a physiological difference in flicker light induced vessel dilation in DM. While the changes of the vascular system are present in all three groups, they can manifest with differing severity and hence partly explain this difference in behaviour.
Further explanations for the differences between groups that may be found in the underlying mechanisms involved in regulating vascular reactivity to flicker light include nitric oxide synthase and bioavailability [32], endothelial function [14] and ganglion cell signalling [33]. 
Table 4 AUC data

\begin{tabular}{|c|c|c|c|c|}
\hline & $\mathrm{DM}(\mathrm{n}=36)$ & CVD $(n=43)$ & DM and CVD $(n=37)$ & $p$ value \\
\hline$A \cup C_{\text {Baseline data }}$ & $-0.9(2.0)$ & $-1.4(2.2)$ & $-1.0(3.1)$ & 0.704 \\
\hline $\mathrm{AUC}_{20 \text { s Flicker }}$ & $14.9(6.0-30.1)$ & $19.8(-0.7-37.8)$ & $12.0(1.1-24.7)$ & 0.429 \\
\hline $\mathrm{AUC}_{20 \text { s Constriction }}$ & $-4.0(-15.7 \text { to } 3.8)^{*}$ & $-14.4(-27.5$ to -3.4$)$ & $-10.1(-20.0$ to -3.7$)$ & 0.038 \\
\hline$A \cup C_{\text {Last } 30 \mathrm{~s}}$ & $-3.5(18.9)$ & $-6.7(26.0)$ & $-7.3(23.1)$ & 0.748 \\
\hline
\end{tabular}

All data are presented as either means ( \pm standard deviation) or median (upper quartile/lower quartile). Statistical significance is shown by bolded $p$-values. All values given in percentage change to baseline vessel diameters or times in seconds

$A U C_{\text {baseline }}$ area under the curve of the averaged $30 \mathrm{~s}$ prior to flicker start, $A U C_{20 \text { s } F L}$ area under the curve of the averaged profile during $20 \mathrm{~s}$ flicker, $A U C_{20}$ Constriction area under the curve of $20 \mathrm{~s}$ duration $10 \mathrm{~s}$ following flicker cessation, $A \cup C_{\text {Last } 30 \mathrm{~s}}$ area under the curve of the last $30 \mathrm{~s}$ of the averaged reation profile

p value by analysis of variance or the Kruskal-Wallis test

${ }^{*} p<0.05$ lower than the two other groups

Table 5 Serum endothelial markers

\begin{tabular}{lccc}
\hline & DM $(\mathbf{n}=\mathbf{3 6})$ & CVD $(\mathbf{n}=\mathbf{4 3})$ & DM and CVD (n= 37) \\
\hline Von Willebrand factor $(\mathrm{IU} / \mathrm{dL})$ & $107(21)^{\mathrm{a}}$ & $111(20)^{\mathrm{b}}$ & $124(22)$ \\
Soluble E selectin $(\mathrm{ng} / \mathrm{mL})$ & $22(8)^{\mathrm{c}}$ & $26(8)$ & $27.5(12)$ \\
\hline
\end{tabular}

Data mean (SD), $\mathrm{p}$ value from ANOVA

a $p=0.004$ to DM and CVD group

b $\mathrm{p}=0.02$ to DM and CVD group

c $p=0.044$ to DM and CVD group (inter-group $p$ values Tukey's post hoc test)

While NO synthase, NO bioavailability and endothelial health can be compromised in both DM and CVD, this is dependent upon disease duration as well as on medication. In this respect our finding of decreased arterial constriction following flicker light provocation in the DM only group is in agreement and expanding the data published by others [17, 34]. One group [33] found that this lack of arterial vasoconstrictor capacity was attributed to an alteration of nitric oxide production and bioavailability, whereas others [17] suggest that the overall reduced dilatory and constriction response could serve as an estimate of endothelial cell capacity to respond to a physiological stimulus. Furthermore, one study examining DM patients with varying severity of retinopathy demonstrated ganglion cell death with increasing severity of the retinopathy [35]. Our finding of more adverse endothelial markers in DM + CVD versus the other groups is in keeping with accepted pathophysiology [36, 37]. However, there were only three instances where levels of the markers correlated meaningfully with an ocular index, suggesting limited predictive value unless confirmed in a far larger population.

Some of the retinal parameters examined in the present study were comparable between the three groups. One explanation for this can be found in the fact that only six diabetic patients suffered from DR. Previous work examining retinal vessel calibres, carotid plaque and the impact of exercise on echocardiography parameters all confirmed that patients with DR compared to those suffering from DM without DR or CVD only had a higher risk of adverse CVD events and impaired myocardial function [38-40]. These findings are somewhat unsurprising given that with increasing duration of DM (and age) the prevalence of DR is increasing too, which is reflecting disease progression and a compromised microcirculation which in turn leads to a higher risk of adverse CVD events. As the majority of the patients assessed in the present study is free from DR this might explain in part why there were only subtle differences across groups. Observations over time taking into consideration DR onset will provide further insight into whether the differences in reaction pattern and the lack of arterial constriction to flicker light provocation in DM only patients is changing over time.

We acknowledge the artificial nature of the three groups, in that it is very likely that many of those we consider to have DM only will be carrying asymptomatic CVD. This also applies to the definition of DM, although we have strength in that our measurement of $\mathrm{HbA}_{1 \mathrm{c}}$ is robust and was performed on all subjects.

\section{Conclusion}

In summary, patients with DM exhibit different retinal vessel reactivity patterns to flicker light provocation compared to those suffering from CVD only or a combination of both. While summarised (i.e. averaged across all flicker 
light cycles) retinal vessel diameters provide only limited capacity to distinguish between groups with different systemic vascular disease, dynamic vessel analysis using SDRA can do so if individual flicker light cycle patterns are analysed. Averaged retinal responses to flicker light lack the capacity to examine a change in reaction pattern and can mask changes in the reaction pattern. We note that only data with three complete cycle recordings should be used for average calculations as it would otherwise potentially distort the dilatory response especially in cases where there are differences between flicker cycles, such as demonstrated in our sample. Future research is needed to establish if retinal vessel reaction patterns change over time or with increased cardiovascular risk factors.

\begin{abstract}
Abbreviations
CVD: cardiovascular disease; DM: diabetes mellitus; NO: nitric oxide; IOP: intraocular pressure; DBP: diastolic blood pressure; SBP: systolic blood pressure; RVA: retinal vessel analyser; CRAE: central retinal artery equivalent; CRVE: central retinal vein equivalent; AVR: artery to vein ratio; BDF: baseline diameter fluctuation; MD: maximum dilation; MC: maximum constriction; DA: dilation amplitude; RT: reaction time; $\mathrm{CT}$ : constriction time; $\mathrm{AUC}_{\text {BASELINE: }}$ area under the curve of the averaged $30 \mathrm{~s}$ prior to flicker start; $\mathrm{AUC}_{20 \mathrm{sF}}$ : area under the curve

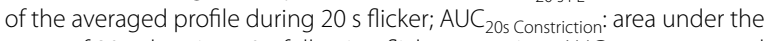
curve of $20 \mathrm{~s}$ duration $10 \mathrm{~s}$ following flicker cessation; $A U C_{\text {last } 30 \text { s: }}$ area under the curve of the last $30 \mathrm{~s}$ of the averaged reaction profile; SDRA: sequential diameter response analyses.
\end{abstract}

\section{Authors' contributions}

$\mathrm{RH}$ and ADB collected and analysed the data and wrote the manuscript. GYHL and RER provided patient access and helped with study design and data interpretation. All authors reviewed the final manuscript. All authors read and approved the final manuscript.

\section{Author details}

${ }^{1}$ School of Life and Health Sciences, Aston University, Aston Triangle, Birmingham B4 7ET, UK. ${ }^{2}$ Institute for Cardiovascular Sciences, University of Birmingham, City Hospital, Birmingham B18 7QH, UK. ${ }^{3}$ Department of Diabetes and Endocrinology, City Hospital, Birmingham B18 7QH, UK.

\section{Acknowledgements}

We would like to thank our colleagues Drs. Chetan Varma, David Nichol, Brian Lee, Jawad Khan and Parijat De for help in recruitment.

\section{Competing interests}

The authors declare that they have no competing interests.

\section{Availability of data and materials}

The datasets used and/or analysed during the current study available from the corresponding author on reasonable request.

\section{Ethics approval and consent to participate}

Ethics approval was granted by the National Health Service Research Ethics Committee East Midlands-Leicester, UK; approval reference: 12/EM/0062.

\section{Funding}

This research was supported by a Hans and Gertrude Hirsch Small Grant from Fight for Sight UK.

\section{Publisher's Note}

Springer Nature remains neutral with regard to jurisdictional claims in published maps and institutional affiliations.
Received: 25 November 2016 Accepted: 11 April 2017

Published online: 26 April 2017

\section{References}

1. Sayin N, Kara N, Pekel G. Ocular complications of diabetes mellitus. World J Diabetes. 2015;6(1):92-108.

2. Szuszkiewicz-Garcia MM, Davidson JA. Cardiovascular disease in diabetes mellitus: risk factors and medical therapy. Endocrinol Metab Clin North Am. 2014;43(1):25-40.

3. Threatt J, Williamson JF, Huynh K, Davis RM. Ocular disease, knowledge and technology applications in patients with diabetes. Am J Med Sci. 2013;345(4):266-70

4. Cheung CY, Ikram MK, Klein R, Wong TY. The clinical implications of recent studies on the structure and function of the retinal microvasculature in diabetes. Diabetologia. 2015;58(5):871-85.

5. Patton N, Aslam T, Macgillivray T, Pattie A, Deary IJ, Dhillon B. Retinal vascular image analysis as a potential screening tool for cerebrovascular disease: a rationale based on homology between cerebral and retinal microvasculatures. J Anat. 2005;206(4):319-48.

6. Liew G, Wang JJ, Mitchell P, Wong TY. Retinal vascular imaging: a new tool in microvascular disease research. Circ Cardiovasc Imaging. 2008;1(2):156-61.

7. Flammer J, Konieczka K, Bruno RM, Virdis A, Flammer AJ, Taddei S. The eye and the heart. Eur Heart J. 2013;34(17):1270-8.

8. Kawasaki R, Cheung N, Mosley T, et al. Retinal microvascular signs and 10-year risk of cerebral atrophy: the Atherosclerosis Risk in Communities (ARIC) study. Stroke. 2010;41(8):1826-8.

9. Kawasaki R, Xie J, Cheung N, et al. Retinal microvascular signs and risk of stroke: the multi-ethnic study of atherosclerosis (MESA). Stroke. 2012;43(12):3245-51.

10. Liew G, Wang JJ, Rochtchina E, Wong TY, Mitchell P. Complete blood count and retinal vessel calibers. PLoS ONE. 2014;9(7):e102230.

11. Wang JJ, Liew G, Wong TY, et al. Retinal vascular calibre and the risk of coronary heart disease-related death. Heart. 2006;92(11):1583-7.

12. von Hanno T, Bertelsen G, Sjølie AK, Mathiesen EB. Retinal vascular calibres are significantly associated with cardiovascular risk factors: the Tromsø Eye Study. Acta Ophthalmol. 2014;92(1):40-6.

13. Ogagarue ER, Lutsey PL, Klein R, Klein BE, Folsom AR. Association of ideal cardiovascular health metrics and retinal microvascular findings: the Atherosclerosis Risk in Communities Study. J Am Heart Assoc. 2013;2(6):e000430.

14. Pemp B, Weigert G, Karl K, et al. Correlation of flicker-induced and flowmediated vasodilatation in patients with endothelial dysfunction and healthy volunteers. Diabetes Care. 2009;32(8):1536-41.

15. Heitmar R, Cubbidge RP, Lip GY, Gherghel D, Blann AD. Altered blood vessel responses in the eye and finger in coronary artery disease. Invest Ophthalmol Vis Sci. 2011;52(9):6199-205.

16. Al-Fiadh AH, Wong TY, Kawasaki $\mathrm{R}$, et al. Usefulness of retinal microvascular endothelial dysfunction as a predictor of coronary artery disease. Am J Cardiol. 2015;115(5):609-13.

17. Mandecka A, Dawczynski J, Blum M, et al. Influence of flickering light on the retinal vessels in diabetic patients. Diabetes Care. 2007;30(12):3048-52.

18. Nguyen TT, Kawasaki R, Kreis AJ, et al. Correlation of light-flicker-induced retinal vasodilation and retinal vascular caliber measurements in diabetes. Invest Ophthalmol Vis Sci. 2009;50(12):5609-13.

19. Lim LS, Ling LH, Ong PG, et al. Dynamic responses in retinal vessel caliber with flicker light stimulation in eyes with diabetic retinopathy. Invest Ophthalmol Vis Sci. 2014;55(8):5207-13.

20. Mishra A, Newman EA. Inhibition of inducible nitric oxide synthase reverses the loss of functional hyperemia in diabetic retinopathy. Glia. 2010;58(16):1996-2004

21. Garhöfer G, Zawinka C, Resch H, Kothy P, Schmetterer L, Dorner GT. Reduced response of retinal vessel diameters to flicker stimulation in patients with diabetes. Br J Ophthalmol. 2004;88(7):887-91.

22. Polak K, Dorner G, Kiss B. Evaluation of the Zeiss retinal vessel analyser. Br J Ophthalmol. 2000;84:1285-90. 
23. Hubbard LD, Brothers RJ, King WN, et al. Methods for evaluation of retinal microvascular abnormalities associated with hypertension/sclerosis in the Atherosclerosis Risk in Communities Study. Ophthalmology. 1999;106(12):2269-80.

24. Blum M, Bachmann K, Wintzer D, Riemer T, Vilser W, Strobel J. Noninvasive measurement of the Bayliss effect in retinal autoregulation. Graefes Arch Clin Exp Ophthalmol. 1999;237:296-300.

25. Seifertl BU, Vilser W. Retinal vessel analyzer (RVA): design and function. Biomed Tech. 2002;47(suppl 1):678-81 (Berl)

26. Garhofer G, Bek T, Boehm AG. Use of the retinal vessel analyzer in ocular blood flow research. Acta Ophthalmol. 2010;88:717-22.

27. Heitmar R, Blann AD, Cubbidge RP, Lip GY, Gherghel D. Continuous retinal vessel diameter measurements: the future in retinal vessel assessment? Invest Ophthalmol Vis Sci. 2010;51(11):5833-9.

28. Gugleta K, Kochkorov A, Waldmann N, et al. Dynamics of retinal vessel response to flicker light in glaucoma patients and ocular hypertensives. Graefes Arch Clin Exp Ophthalmol. 2012;250(4):589-94.

29. Kotliar KE, Lanzl IM, Schmidt-Trucksäss A, et al. Dynamic retinal vessel response to flicker in obesity: a methodological approach. Microvasc Res. 2011:81(1):123-8.

30. Lott ME, Slocomb JE, Shivkumar V, et al. Comparison of retinal vasodilator and constrictor responses in type 2 diabetes. Acta Ophthalmol. 2012;90(6):e434-41.

31. Lott ME, Slocomb JE, Gao Z, et al. Impaired coronary and retinal vasomotor function to hyperoxia in individuals with type 2 diabetes. Microvasc Res. 2015;101:1-7.
32. Dorner GT, Garhofer G, Kiss B, et al. Nitric oxide regulates retinal vascular tone in humans. Am J Physiol Heart Circ Physiol. 2003;285(2):H631-6.

33. Newman EA. Glial cell regulation of neuronal activity and blood flow in the retina by release of gliotransmitters. Phil Trans R Soc. 2015; B 370:20140195.

34. Lott ME, Slocomb JE, Shivkumar V, et al. Impaired retinal vasodilator responses in prediabetes and type 2 diabetes. Acta Ophthalmol. 2013;91(6):e462-9.

35. Adamiec-Mroczek J, Zając-Pytrus H, Misiuk-Hojło M. Caspase-dependent apoptosis of retinal ganglion cells during the development of diabetic retinopathy. Adv Clin Exp Med. 2015;24(3):531-5.

36. Seidelmann SB, Claggett B, Bravo PE, et al. Retinal vessel calibers in predicting long-term cardiovascular outcomes: the atherosclerosis risk in communities study. Circulation. 2016;134(18):1328-38.

37. Blann AD. Plasma von Willebrand factor, thrombosis, and the endothelium: the first 30 years. Thromb Haemost. 2006;95(1):49-55.

38. Roldán V, Marín F, Lip GY, Blann AD. Soluble E-selectin in cardiovascular disease and its risk factors. A review of the literature. Thromb Haemost. 2003;90(6):1007-20.

39. Alonso N, Traveset A, Rubinat E, et al. Type 2 diabetes-associated carotid plaque burden is increased in patients with retinopathy compared to those without retinopathy. Cardiovasc Diabetol. 2015;14:33.

40. Zhen Z, Chen Y, Shih K, et al. Altered myocardial response in patients with diabetic retinopathy: an exercise echocardiography study. Cardiovasc Diabetol. 2015;14:123

\section{Submit your next manuscript to BioMed Central and we will help you at every step:}

- We accept pre-submission inquiries

- Our selector tool helps you to find the most relevant journal

- We provide round the clock customer support

- Convenient online submission

- Thorough peer review

- Inclusion in PubMed and all major indexing services

- Maximum visibility for your research

Submit your manuscript at www.biomedcentral.com/submit 